\title{
Experiencia con la dieta cetogénica como tratamiento en la epilepsia refractaria
}

\author{
Alia Ramírez-Camacho, Silvia Meavilla, Natalia Catalán, Alejandra Gutiérrez, Jaume Campistol
}

Servicio de Neurología (A. RamírezCamacho, J. Campistol); Sección de Gastroenterología y Nutrición (S. Meavilla, N. Catalán, A. Gutiérrez); Hospital Sant Joan de Déu Universitat de Barcelona. Esplugues de Llobregat, Barcelona, España.

Correspondencia: Dr. Jaume Campistol Plana. Servicio de Neurología. Hospital Sant Joan de Déu. Passeig Sant Joan de Déu 2. E-08950 Esplugues de Llobregat (Barcelona).

E-mail:

campistol@hsjdbcn.org

Aceptado tras revisión externa: 07.04.11.

Cómo citar este artículo: Ramírez-Camacho A, Meavilla S, Catalán N, Gutiérrez A, Campistol J. Experiencia con la dieta cetogénica como tratamiento en la epilepsia refractaria. Rev Neurol 2011; 53: $524-30$

(c) 2011 Revista de Neurología

\section{Introducción}

La epilepsia es una enfermedad en la que -en la mayoría de los casos- se logra un adecuado control con los fármacos antiepilépticos (FAE). Sin embargo, aproximadamente en un $25 \%$ de casos la epilepsia es refractaria al tratamiento farmacológico [1].

La dieta cetogénica se ha utilizado como tratamiento anticonvulsivo desde 1921. Con la aparición de los FAE su uso disminuyó; sin embargo, en los últimos 20 años ha vuelto a resurgir como tratamiento de la epilepsia refractaria a medida que se realizan más investigaciones científicas acerca de su efectividad y conforme no se obtienen los resultados esperados con los nuevos FAE [2,3].

Hasta el momento, no se conoce el mecanismo de acción específico por el cual la dieta cetogénica tiene un efecto anticonvulsivo. La investigación realizada en torno a ella sugiere múltiples mecanismos de acción en neurotransmisores, metabólicos y genéticos [4]. Hay evidencia que defiende que la dieta cetogénica favorece la síntesis de glutamina, precursor esencial del ácido $\gamma$-aminobutírico, que es un neurotransmisor inhibitorio y un importante agente anticonvulsivo. Otros estudios sugieren entre los posibles mecanismos de acción que la dieta también altera el metabolismo o la función de las aminas biógenas $[4,5]$.

Se postuló que la dieta cetogénica tenía la misma efectividad en los distintos tipos de epilepsia. Actualmente la evidencia parece demostrar un mayor beneficio en ciertos tipos de epilepsia: mioclónica atónica, síndrome de Dravet y displasias corticales, entre otras $[3,6]$. En dos entidades metabólicas, deficiencia del transportador de glucosa cerebral tipo 1 (GLUT-1) y en la deficiencia de la piruvato deshidrogenasa (especialmente en la primera), se considera el tratamiento de primera elección [2,3].

Se utilizan diferentes tipos de dieta cetogénica de acuerdo con los hábitos alimentarios y los requerimientos nutricionales de los pacientes además de la experiencia del centro hospitalario donde se administre [6,7]. Existen variaciones en las relaciones entre lípidos y carbohidratos + proteínas (4 a 1 o 3 a 1), así como en la fuente lipídica (triglicéridos de cadena larga o media) de la dieta cetogénica, sin 
encontrarse diferencias en su efectividad, aunque sí en su tolerancia $[8,9]$ (Tabla I).

En la dieta cetogénica clásica el $90 \%$ de la energía total procede de las grasas saturadas de cadena larga de los alimentos, o de un preparado comercial especial para dieta cetogénica. Su relación con el contenido en hidratos de carbono y proteínas puede variar, lo que da lugar a ratios de 3 a 1 o 4 a 1 [10-12] (es decir, por cada 3 o $4 \mathrm{~g}$ de grasa se administra $1 \mathrm{~g}$ de hidratos de carbono más proteínas). El inconveniente de esta dieta es que el exceso de grasa la hace menos apetecible y poco variada [10-13].

En la dieta cetogénica con triglicéridos de cadena media (TCM) se añade este suplemento de aceite hasta alcanzar un $60 \%$ del total de las calorías, con lo que se disminuye así el total de grasas procedentes de la dieta. Esta dieta tiene mayor capacidad para inducir cetosis e incluye menor proporción de grasas, pero se ha visto una mayor intolerancia digestiva en aceites ricos en TCM con aparición de diarreas frecuentes, lo que la hace menos tolerable que la dieta cetogénica clásica [14].

La dieta cetogénica combinada está constituida por un $30 \%$ de TCM y un $40 \%$ de grasas de cadena larga, con lo que las grasas suponen un $70 \%$ del total de la energía, de modo que se mejora la tolerancia de la dieta, haciéndola más apetecible, a diferencia de los otros tipos de dieta. Sin embargo, no ha demostrado ser más efectiva que las anteriores [12,13].

La dieta cetogénica modificada incorpora grasas de cadena media y larga, con menores volúmenes de aceite, lo que la vuelve más apetecible. Ésta tampoco ha demostrado ser más efectiva que las anteriores. Otra alternativa a la dieta cetógena es la dieta Atkins [15], que también induce a la cetosis, es menos restrictiva en calorías, proteínas y líquidos y no requiere el ingreso hospitalario en la implantación de la dieta. La dieta Atkins consiste en un $60 \%$ del valor calórico total en forma de grasas, un $30 \%$ del valor calórico total en forma de proteínas y un aporte inicial de $10 \mathrm{~g}$ /día de carbohidratos; además se puede modificar según el estado del paciente. Este tipo de dieta se debe suplementar con complejos vitamínicos y minerales. Se ha observado que provoca menos efectos adversos, y es mejor tolerada por el paciente, ya que disminuye los episodios de náuseas, vómitos y pérdida de apetito. Una de las desventajas de ésta es que la inducción a la cetosis resulta más lenta en comparación con la dieta cetogénica $[15,16]$.

Existen algunas recomendaciones para el uso de la dieta cetogénica como tratamiento en la epilepsia refractaria (Tabla II). Sin embargo, a pesar del aumento en los últimos años de la popularidad de di-
Tabla I. Composición de las distintas variantes de dieta cetogénica.

\begin{tabular}{lcccc}
\hline & Grasas & $\begin{array}{c}\text { Triglicéridos de } \\
\text { cadena media }\end{array}$ & Proteínas & Carbohidratos \\
\hline Clásica 3 a 1 & $87 \%$ & - & $13 \%$ & Entre ambas \\
\hline Clásica 4 a 1 & $90 \%$ & - & $10 \%$ & Entre ambas \\
\hline Triglicéridos de cadena media & $11 \%$ & $60 \%$ & $11 \%$ & $19 \%$ \\
\hline Combinada & $40 \%$ & $30 \%$ & $10 \%$ & $20 \%$ \\
\hline
\end{tabular}

cha dieta como tratamiento anticonvulsionante, no se ha establecido un consenso universal para sus indicaciones y manejo.

El objetivo fue evaluar la respuesta, tolerancia y efectos secundarios en los pacientes con epilepsias refractarias que han recibido dieta cetogénica en nuestra institución en un período de 20 años.

\section{Pacientes y métodos}

Se revisaron las historias clínicas de los pacientes en seguimiento por el Servicio de Neurología y la Sección de Gastroenterología y Nutrición del Hospital Sant Joan de Déu, que utilizaron la dieta cetogénica como tratamiento coadyuvante de la epilepsia refractaria en los últimos 20 años.

La muestra estaba constituida por 30 pacientes: 14 mujeres y 16 varones. La edad de inicio de la epilepsia fue entre los 3 días de vida y los 6 años de edad, con una edad media de 14,8 meses. La edad en el momento del inicio de la dieta cetogénica fue entre los 6 meses y 15 años, con una edad media de 32,3 meses. Se excluyeron tres pacientes de sexo masculino por datos incompletos en la historia clínica para obtener la información necesaria, por lo que finalmente se incluyeron 27 pacientes.

Los diagnósticos de los diferentes tipos de epilepsia se basaron en la última clasificación de la Liga Internacional contra la Epilepsia (ILAE) $[17,18]$. La distribución de acuerdo con ésta fue la siguiente: epilepsia focal en 8 casos $(29,6 \%)$, epilepsia generalizada en $10(37,3 \%)$ y encefalopatías epilépticas en 9 (33,3\%).

Se consideró epilepsia refractaria aquélla en la que las crisis no se lograron controlar mediante el tratamiento adecuado con dos FAE tolerados, adecuadamente elegidos y pautados [17]. Todos los pacientes de nuestra muestra cumplían con las carac- 
Tabla II. Indicaciones de la dieta cetogénica en la epilepsia refractaria.

\begin{tabular}{|c|c|}
\hline Tratamiento de elección & $\begin{array}{l}\text { Déficit del transportador } \\
\text { de glucosa cerebral (GLUT-1) }\end{array}$ \\
\hline \multirow{6}{*}{$\begin{array}{l}\text { Tratamiento } \\
\text { probablemente útil }\end{array}$} & Síndrome de Doose \\
\hline & Síndrome de Dravet \\
\hline & Síndrome de Lennox-Gastaut \\
\hline & Espasmos infantiles \\
\hline & Esclerosis tuberosa \\
\hline & Síndrome de Rett \\
\hline \multirow{2}{*}{ Tratamiento en estudio } & Punta-onda continua del sueño \\
\hline & Estado de mal epiléptico \\
\hline
\end{tabular}

terísticas de esta definición, habiendo recibido como tratamiento entre tres y seis FAE en monoterapia o terapia combinada. Todos los pacientes presentaban más de cuatro crisis semanales de acuerdo con el registro de éstas, por parte de la familia, previo al inicio de la dieta.

En ningún caso se había utilizado la administración de inmunoglobulinas, estimulación del nervio vago o cirugía como terapia antiepiléptica previamente a la dieta cetogénica. Sólo dos pacientes recibieron esteroides antes de la dieta, sin éxito.

El tipo de dieta se indicó en función de las características del paciente y de la tolerancia al tipo de dieta (clásica, con TCM o combinada).

La eficacia de la dieta cetogénica se evaluó de acuerdo con el porcentaje de reducción de la frecuencia de las crisis (> 75\%, 74-50\% o < 50\%). Se consideró una respuesta positiva cuando los pacientes presentaron una reducción $>50 \%$ de las crisis de base (Tabla III). El plazo mínimo para valorar la efectividad de la dieta fue de tres meses. Si no se obtenía una respuesta favorable a partir de los tres meses, se retiraba la dieta. Todos los pacientes, a excepción de dos, iniciaron la dieta cetogénica en nuestro centro durante un ingreso programado de 3-4 días. Durante dicho ingreso se explicó a la familia la pauta dietética, que se individualizó hasta obtener una cetonuria adecuada, modificándola según la tolerancia.

Se consideraron efectos secundarios a corto plazo los que aparecieron dentro de las primeras cuatro semanas de instauración de la dieta cetogénica, y como efectos secundarios a largo plazo, aquellos que se presentaron con posterioridad a este período. Éstos se monitorizaron según el protocolo establecido en nuestra institución mediante control nutricional -con valoración antropométrica y velocidad de crecimiento- y controles bioquímicos cada tres meses, ecografía renal, control oftalmológico y electrocardiograma cada seis meses $[11,12]$.

\section{Resultados}

Los pacientes recibieron distintos tipos de dieta cetogénica según las características de cada caso: 9 pacientes recibieron una dieta cetogénica clásica; 6 pacientes, una dieta combinada, y 12, una dieta con TCM. Se alcanzó una cetonuria adecuada (igual o mayor $\mathrm{a}+++)$ solamente en 15 pacientes $(55,5 \%)$, y de éstos sólo 10 obtuvieron un respuesta positiva anticonvulsiva con una reducción $>50 \%$ de las crisis.

El cumplimiento de la dieta por parte del paciente y sus familiares fue correcto a excepción de dos casos. En estos dos casos no se alcanzó una adecuada cetonuria en ningún momento del tiempo establecido para valorar su efectividad y, finalmente, abandonaron la dieta.

Del total de pacientes $(n=27)$ con dieta cetogénica, 10 (37\%) presentaron una respuesta positiva en la reducción del número de crisis por más de seis meses; cinco de ellos con una disminución del 50$75 \%$, y cinco $>75 \%$. Los pacientes que mostraron una respuesta positiva mantuvieron la dieta cetogénica entre 19 meses y 6 años según los resultados obtenidos, sin agravamiento de su epilepsia durante el tratamiento con la dieta ni tras su suspensión.

Todos los pacientes presentaban al inicio de la dieta cetogénica politerapia con un mínimo tres FAE, por lo que se consideraron pacientes con epilepsia refractaria. En cinco pacientes, dada la estabilidad en el control de crisis con la dieta cetogénica, se logró disminuir el número de FAE empleados y se pasó de un promedio de dos FAE a un solo FAE.

Sólo se observaron cambios electroencefalográficos favorables y mantenidos en los pacientes que ofrecieron una respuesta positiva en la reducción de las crisis $>75 \%$. En estos casos se constató una mejoría en el trazado de base, así como la desaparición de las anomalías paroxísticas. Lógicamente, la mejoría de las crisis coincide con la mejoría electroencefalográfica en los casos respondedores.

Los pacientes mantuvieron el tratamiento con dieta cetogénica un mínimo de tres meses para valorar su efectividad. Sólo en siete pacientes se debió suspender la dieta cetogénica antes del mes por in- 
Tabla III. Resultados de la dieta cetogénica en función del síndrome epiléptico.

\begin{tabular}{|c|c|c|c|c|c|c|}
\hline & \multirow{2}{*}{$\begin{array}{l}\text { Síndrome electroclínico } \\
\text { y otros tipos de epilepsia }\end{array}$} & \multirow{2}{*}{$\begin{array}{c}\mathrm{N} . \mathrm{o} \text { de } \\
\text { observaciones }\end{array}$} & \multicolumn{4}{|c|}{ Reducción del número de crisis } \\
\hline & & & $>75 \%$ & $74-50 \%$ & $<50 \%$ & Sin respuesta \\
\hline \multirow{7}{*}{$\begin{array}{l}\text { Convulsiones } \\
\text { generalizadas }\end{array}$} & $\begin{array}{l}\text { Epilepsia secundaria a } \\
\text { cromosoma } 20 \text { en anillo }\end{array}$ & 1 & & 1 & & \\
\hline & Epilepsia mioclónica idiopática & 1 & & & & 1 \\
\hline & Epilepsia refleja & 1 & & & & 1 \\
\hline & Epilepsia secundaria a meningitis & 1 & & & & 1 \\
\hline & Epilepsia tónica generalizada & 1 & & & & 1 \\
\hline & Epilepsia secundaria a malformación cortical & 3 & & 1 & & 2 \\
\hline & Epilepsia secundaria a déficit de GLUT-1 & 2 & 2 & & & \\
\hline \multirow{5}{*}{$\begin{array}{l}\text { Convulsiones } \\
\text { focales }\end{array}$} & Esclerosis tuberosa & 1 & & & & 1 \\
\hline & Epilepsia secundaria a cavernoma & 1 & & & & 1 \\
\hline & Epilepsia secundaria a displasia cortical & 2 & & & & 2 \\
\hline & Epilepsia focal frontal & 1 & & & & 1 \\
\hline & Epilepsia secundaria a déficit de GLUT-1 & 3 & 2 & 1 & & \\
\hline \multirow{6}{*}{$\begin{array}{l}\text { Encefalopatías } \\
\text { epilépticas }\end{array}$} & Síndrome de Ohtahara & 2 & & 1 & & 1 \\
\hline & Síndrome de West & 2 & & & & 2 \\
\hline & Síndrome de Lennox-Gastaut & 1 & & & & 1 \\
\hline & Síndrome de Dravet & 1 & & & & 1 \\
\hline & Síndrome de Doose & 2 & 1 & 1 & & \\
\hline & Epilepsia con mutación en CDKL5 & 1 & & & & 1 \\
\hline
\end{tabular}

tolerancia o efectos secundarios a corto plazo. Los vómitos fueron la principal causa de intolerancia.

La tolerancia de la dieta fue aceptable en la mayoría de los casos, a excepción de los siete pacientes $(25,9 \%)$ ya mencionados. Cinco pacientes presentaron vómitos continuos, y dos, hipoglucemias, a pesar del ajuste del aporte calórico y de la fuente lipídica de la dieta. No observamos una mejor tolerancia con un tipo de dieta específica.

Los efectos adversos a corto plazo que evidenciaron nuestros pacientes fueron diarrea en 13 pa- cientes $(48,1 \%)$, vómitos en $8(29,6 \%)$, hipoglucemias en $6(22,2 \%)$, acidosis metabólica en $1(3,7 \%)$, incremento de enzimas hepáticas en $1(3,7 \%)$ y aumento del colesterol en 1 (3,7\%), efectos que no obligaron a suspender la dieta y que se corrigieron con reajustes dietéticos.

Los efectos adversos observados a largo plazo fueron estreñimiento en 4 pacientes $(14,8 \%)$, aumento de peso en $3(11,1 \%)$, enlentecimiento del crecimiento en $1(3,7 \%)$, deficiencia de zinc, hierro y calcio en $1(3,7 \%)$, y aumento del colesterol en $1(3,7 \%)$. 


\section{Discusión}

En nuestra experiencia, la respuesta positiva en la reducción del numero de crisis ha sido algo menor que la publicada en otros estudios $[13,16,19,20]$, en los que se comunica una disminución $>50 \%$ de la frecuencia de las crisis en un $60 \%$ de los pacientes con dieta cetogénica. Cabe señalar que la población de nuestra muestra es pequeña y que en menos de la mitad de los pacientes se alcanzó una cetonuria adecuada. Además, la mayoría de los pacientes iniciaron la dieta cetogénica algo tarde, después de haber utilizado más de cinco FAE. En otros trabajos se recomienda iniciarla tras haber ensayado dos FAE o antes, para lograr mayor efectividad $[16,19$, 20]. Los pacientes que presentaron una mayor disminución del número de convulsiones fueron aquellos con epilepsia secundaria a deficiencia de GLUT-1. En el grupo de las encefalopatías epilépticas, el síndrome de Doose y el síndrome de Ohtahara fueron los que alcanzaron una reducción en el número de convulsiones del 50-74\%, sin objetivar disminución alguna en los dos pacientes con síndrome de West ni en los otros síndromes epilépticos. Sin embargo, la serie es muy corta para extraer conclusiones.

En nuestra muestra no se encontró una mayor efectividad ni tampoco una mayor tolerancia a un tipo de dieta específico, como se menciona en la bibliografía [16,20,21]. Al igual que los tratamientos farmacológicos anticonvulsivos, la dieta cetogénica presenta varios efectos adversos. Una de las ventajas con respecto a algunos FAE es que no se han evidenciado efectos secundarios cognitivos. Sin embargo, la mayoría de los estudios realizados no valoran los que puedan presentar los pacientes años después de haber recibido la dieta cetogénica, sino los que se presentan durante la utilización de ésta $[20,22]$. En nuestra población no hallamos efectos secundarios cognitivos a largo plazo, ni litiasis renal, ni afectación hepática. El paciente que mayor tiempo ha mantenido la dieta (seis años) se encuentra libre de crisis y asintomático, de acuerdo con los controles realizados por nuestros servicios. En este paciente afecto de epilepsia mioclónica atónica hemos logrado la remisión completa de las crisis epilépticas, una mejoría electroencefalográfica y en el rendimiento escolar y la reducción del tratamiento con FAE; se pasó de tres fármacos a monoterapia.

En la bibliografía se mencionan efectos secundarios casi inmediatos a la introducción de la dieta (vómitos, cálculos renales, hemorragias gastrointestinales, hiperlipidemia tipo I, colitis ulcerosa e incluso coma) $[6,7,21]$. También se citan alteraciones bioquímicas precoces tras la instauración de la dieta cetogénica: hipoglucemia, hipertrigliceridemia, hiperuricemia, hipertransaminemia, hipercolesterolemia, hipoproteinemia, hipomagnesemia, hiponatremia y acidosis metabólica $[8,19,20]$. En algunas ocasiones hemos constatado la hipoglucemia, que se resolvió con medidas dietéticas.

A largo plazo también se han comunicado efectos negativos de la dieta, como retardo del desarrollo, fallo hepático, estreñimiento, exacerbación, reflujo gastroesofágico, urolitiasis, osteopenia, cardiomiopatía, alargamiento del intervalo QT, neuropatía óptica y alteraciones en los ganglios basales [22,23].

Las complicaciones bioquímicas a largo plazo de la dieta cetogénica son anemia, hipocarnitinemia, dislipidemias, elevación de ácidos grasos de cadena muy larga y déficits vitamínicos y minerales [24].

Existe además una serie de patologías en las que la introducción de la dieta cetogénica podría ser contraproducente y podría agravar la situación basal, como la acidosis metabólica, la deshidratación, la presencia de cálculos renales, cardiomiopatía, hepatitis, pancreatitis y problemas gastrointestinales. También se debería tener precaución cuando el paciente recibe FAE (zonisamida, topiramato o acetazolamida) que producen acidosis metabólica [23,24].

Nuestros resultados son similares; sin embargo, tenemos la impresión de que cuando se instaura lentamente la dieta con una buena explicación y aceptación por parte de la familia y del niño, la tolerancia y los resultados son mejores. Los efectos adversos, si bien son numerosos, son tolerables y, en general, transitorios. Un equipo multidisciplinar que conozca bien la dieta cetogénica y sus complicaciones puede ayudar a minimizar los posibles efectos secundarios precoces y anticiparse a las complicaciones tardías [12,21,22]. Es también importante seleccionar a los pacientes candidatos a la dieta aun a pesar de que no existen criterios universales de tratamiento. Cabe mencionar que los efectos secundarios a corto y largo plazo que observamos en la serie se detectaron durante el seguimiento controlado de nuestros pacientes y se corrigieron sin presentar mayores complicaciones hasta el momento de realización de este estudio. Es de gran importancia el control y manejo nutricional de estos pacientes. Con pequeñas modificaciones en la dieta y el aporte de suplementos minerales, como se señala en la bibliografía [22-24], se eliminaron las posibles complicaciones atribuidas a la dieta.

En los estudios realizados sobre la dieta cetogénica se revela un efecto favorable en la epilepsia [7, $25,26]$. Sin embargo, la mayoría de los estudios son observacionales y carecen de validez estadística. En una revisión Cochrane se concluye que no hay evi- 
dencia científica en estudios aleatorizados controlados sobre la dieta cetogénica, aunque se considera una opción más en el tratamiento de las epilepsias refractarias [6]. En el 2008 se publicaron los resultados del primer estudio aleatorizado controlado, donde se observa la eficacia de la dieta cetogénica como tratamiento de las epilepsias refractarias [7].

Debemos señalar el valor de la dieta cetogénica en los pacientes con defecto del GLUT-1, que con mucha frecuencia asocia epilepsia refractaria a los FAE. El uso precoz de la dieta permite controlar la epilepsia y mejorar el pronóstico de estos pacientes. En otras enfermedades metabólicas también se preconiza el empleo de la dieta cetogénica; no obstante, los resultados no son tan espectaculares como en el GLUT-1. Por contra, en algunas enfermedades metabólicas la dieta cetogénica puede empeorar la situación basal (porfiria, acidurias orgánicas, déficits de piruvato carboxilasa, defectos de oxidación de ácidos grasos, defectos primarios de la cetogénesis/ cetólisis, etc. $[2,8,12,21]$.

Finalmente, como se expone en la tabla II, se está ensayando la dieta cetogénica en otros tipos de epilepsia, e incluso en el estado de mal epiléptico.

En conclusión, a pesar de las limitaciones de nuestro estudio -retrospectivo y con una muestra corta de pacientes-, podemos observar que existe una respuesta positiva en el control de las crisis epilépticas con la dieta cetogénica en más de un tercio de los casos de epilepsia refractaria. La dieta cetogénica puede ser efectiva, pero no está exenta de riesgos. Los efectos adversos deben conocerse: un nutricionista con experiencia puede prevenirlos o corregirlos sin mayores complicaciones. Es necesario estandarizar las indicaciones específicas y el manejo de la dieta cetogénica para poder llevar a cabo estudios con validez suficiente como para demostrar su efectividad. Es probable que con una mejor monitorización de la dieta cetogénica se puedan alcanzar mejores resultados si se consigue introducir y tolerar adecuadamente.

Con los datos comunicados, la dieta cetogénica es una opción más en el tratamiento de las epilepsias refractarias antes de valorar otras opciones terapéuticas más agresivas, como la colocación de un estimulador del nervio vago u otro tipo de cirugía, o incluso en pacientes que no sean candidatos a cirugía. Además está muy especialmente indicada en el déficit del GLUT-1.

\section{Bibliografía}

1. Sevilla-Castillo RA, Palacios GC, Ramírez-Campos J, MoraPuga M, Díaz-Bustos R. Methylprednisolone for the treatment of children with refractory epilepsy. Neuropediatrics 2009; 40: $265-8$.

2. Barañano KW, Hartman AL. The ketogenic diet: uses in epilepsy and other neurologic illnesses. Curr Treat Options Neurol 2008; 10: 410-9.

3. Kossoff EH, Zupec-Kania BA, Amark PE, Ballaban-Gil KR, Bergqvist AGC, Blackford R, et al. Optimal clinical management of children receiving the ketogenic diet: recommendations of the International Ketogenic Diet Study Group. Epilepsia 2009; 50: 304-17.

4. Bough KJ, Rho JM. Anticonvulsant mechanisms of the ketogenic diet. Epilepsia 2007; 48: 43-58.

5. Yudkoff M, Daikhin Y, Melo TM, Nissim I, Sonnewald U, Nissim I. The ketogenic diet and brain metabolism of amino acids: relationship to the anticonvulsant effect. Ann Rev Nutr 2007; 27: 415-30.

6. Levy RG, Cooper PP. Ketogenic diet for epilepsy. The Cochrane Collaboration 2010; 1: 1-6.

7. Neal EG, Chaffe H, Schwartz RH, Lawson MS, Edwards N, Fitzsimmons G, et al. The ketogenic diet for the treatment of childhood epilepsy: a randomised controlled trial. Lancet Neurol 2008; 7: 500-6.

8. Porta $\mathrm{N}$, Vallée L, Boutry $\mathrm{E}$, Auvin $\mathrm{S}$. Le régime cétogène et ses variantes: certitudes et doutes. Rev Neurol (Paris) 2009; 165: 430-9.

9. Moreno-Villares JM, Oliveros-Leal L, Simón-Heras R, Mateos-Beato F. La vuelta a la dieta cetogénica. ¿Qué papel desempeña en el tratamiento de las convulsiones infantiles refractarias? Rev Neurol 2001; 32: 1115-9.

10. Levy R, Cooper P. Ketogenic diet for epilepsy. Cochrane Database Syst Rev 2003; 3: CD001903.

11. Freeman JM. What every pediatrician should know about the ketogenic diet. Contemp Pediatr 2003; 20: 113-27.

12. Galván-Manso M, Arellano M, Sans A, Sanmartí FX, Gómez L, Vernet A, et al. Dieta cetogénica: i una alternativa válida en epilepsias refractarias? Rev Neurol 2001; 33: 1-5.

13. Thiele EA. Assessing the efficacy of antiepileptic treatments: the ketogenic diet. Epilepsia 2003; 44: 26-9.

14. Panico LR, De Martini MG, Ríos VG, Carniello MA. Dieta cetogénica en la epilepsia refractaria infantil: respuesta electroclínica, complicaciones y efectos secundarios. Rev Neurol 2000; 31: 212-20.

15. Tonekabone SH, Mostaghimi P, Mirmiran P, Abbaskhanian A, Abdollah F, Gorji F, et al. Efficacy of the Atkins diet as therapy for intractable epilepsy in children. Arch Iran Med 2010; 13: 492-7.

16. Auvin S, Porta N, Pourrat M, Bellavoine V, Vallée L. Évolution de la prescription du régime cétogène en France chez l'enfant entre 2001 et 2008. Rev Neurol (Paris) 2010; 166: 314-20.

17. Kwan P, Arzimanoglou A, Berg AT, Brodie MJ, Hauser WA, Mathern G, et al. Definition of drug resistant epilepsy: consensus proposal by the ad hoc Task Force of the ILAE Commission on Therapeutic Strategies. Epilepsia 2010; 51 : 1069-77.

18. Berg AT, Berkovic SF, Brodie MJ, Buchhalter J, Cross JH, Boas W, et al. Revised terminology and concepts for organization of seizures and epilepsies: report of the ILAE Commission on Classification and Terminology, 2005-2009. Epilepsia 2010; 51: 676-85.

19. Coppola G, Veggiotti A, Ammendola E, Operto FF, Della Corte R, Signoriello G, et al. Ketogenic diet for the treatment of catastrophic epileptic encephalopathies in childhood. Eur J Paediatr Neurol 2010; 14: 229-34

20. Beniczky S, Miranda MJ, Alving J, Povlsen JH, Wolf P. Effectiveness of the ketogenic diet in a broad range of seizures types and EEG features for severe childhood epilepsies. Acta Neurol Scand 2010; 121: 58-62.

21. Kossoff EH, Zupec-Kania BA, Rho JM. Ketogenic diets: an update for child neurologists. J Child Neurol 2009; 24: 979-88.

22. Patel A, Pyzik PA, Turner Z, Rubenstein JE, Kossoff EH. Long-term outcomes of children treated with the ketogenic diet in the past. Epilepsia 2010; 51: 1277-82.

23. Evangeliou A, Spilioti M, Doulioglou V, Kalaidopoulou P, 
Ilias A, Skarpalezou A, et al. Branched chain amino acids as adjunctive therapy to ketogenic diet in epilepsy: pilot study and hypothesis. J Child Neurol 2009; 24: 1268-72.

24. Kang HC, Chung DE, Kim DW, Kim HD. Early- and late-onset complications of the ketogenic diet for intractable epilepsy. Epilepsia 2004; 45: 1116-23.

25. Van Delft R, Lambrechts D, Verschuure P, Hulsman J, Majoie M.
Blood beta-hydroxybutyrate correlates better with seizure reduction due to ketogenic diet than do ketones in the urine. Seizure 2010; 19: 36- 9.

26. Amari A, Dahlquist L, Kossoff EH, Vining EPG, Trescher WH, Slifer KJ. Children with seizures exhibit preferences for foods compatible with the ketogenic diet. Epilepsy Behav 2007; 11 98-104.

\section{Experience with ketogenic diet as treatment for refractory epilepsy}

Introduction. Epilepsy is a disease where most patients have a good control with pharmacological antiepileptic treatment. Nevertheless, $25 \%$ of the patients have a refractory epilepsy to usual antiepileptic drugs. Ketogenic diet is one of the treatment options for this type of epilepsy. In spite of the increased popularity of it as an antiepileptic treatment, it does not exist an international consensus of its indications and management.

Aim. To evaluate the response, tolerance and adverse effects of the patients with refractory epilepsy at our hospital during the last 20 years.

Patients and methods. We reviewed the data of 30 patients with ketogenic diet and the follow-up at the Neurology and Nutrition Services in our Hospital.

Results. Ten patients (35.7\%) had a positive response with reduction of their seizures for more than six months; five of them had a $50-75 \%$ decrease in seizures and five of them had more than $75 \%$ of seizure reduction. The most common short term adverse effects were diarrhea, vomiting and hypoglicemia whereas long term adverse effects were constipation and weight gain.

Conclusions. We recommend to use ketogenic diet as treatment in refractory epilepsy since there is a positive response in seizure control in some cases. The adverse effects seen could be prevented or treated without complications. It is a preferable treatment option before using other aggressive therapeutical measures or when surgery is not feasible.

Key words. Adverse effects. Epileptic encephalopathy. Ketogenic diet. Refractory epilepsy. 